\title{
Vivre dans deux temps
}

Les calendriers lunaires des Juifs italiens à l'heure de l'émancipation (XVIII ${ }^{\mathrm{e}}-\mathrm{XX}$ siècles)

Living in Two Times. The Lunar Calendars of Italian Jews at the Time of Emancipation (18 $8^{\text {th }}-20^{\text {th }}$ Centuries)

\section{Davide Mano}

\section{CpenEdition}

\section{Journals}

Édition électronique

URL : https://journals.openedition.org/cher/4379

DOI : $10.4000 /$ cher. 4379

ISSN : 2803-5992

\section{Éditeur}

Presses universitaires de Strasbourg

\section{Édition imprimée}

Date de publication : 30 novembre 2021

Pagination : $97-114$

ISBN : 979-10-344-0095-9

ISSN : 1968-035X

\section{Référence électronique}

Davide Mano, «Vivre dans deux temps », reCHERches [En ligne], 27 | 2021, mis en ligne le 30 novembre 2021, consulté le 12 janvier 2022. URL : http://journals.openedition.org/cher/4379 ; DOI : https:// doi.org/10.4000/cher.4379

\section{(2) $\odot \Theta \odot$}

$\mathrm{Ce}(\mathrm{tte})$ œuvre est mise à disposition selon les termes de la Licence Creative Commons Attribution -

Pas d'Utilisation Commerciale - Partage dans les Mêmes Conditions 4.0 International. 


\title{
Vivre dans deux temps Les calendriers lunaires des Juifs italiens à l'heure de l'émancipation (XVIII'-XXe siècles)
}

\author{
DaVIDE MAN0*
}

\begin{abstract}
$\mathrm{D}$ ans cet article, j'entends présenter les premiers résultats d'une recherche en cours sur une documentation extrêmement riche et historiquement intéressante, rédigée en italien et en hébreu: les calendriers lunaires juifs publiés en Italie entre le XVIII ${ }^{e}$ siècle et le $\mathrm{xx}^{\mathrm{e}}$ siècle. Outils d'usage quotidien reliant le travail agricole à la vie communautaire, les calendriers ou almanachs juifs ont fonctionné - et fonctionnent encore aujourd'hui - en tant qu'instruments pour l'éducation religieuse et politique, ainsi que pour la vulgarisation culturelle et scientifique. Si la littérature consacrée à l'expression du temps dans le judaïsme est abondante (Goldberg 2000 et 2004, Carlebach 2011, Laras 2015), l'étude des calendriers lunaires en tant qu'objets de la culture populaire reste encore en grande partie à faire ${ }^{1}$.
\end{abstract}

Cet article propose une première analyse sur un échantillon constitué d'une centaine de calendriers lunaires juifs publiés à Turin, Casale Monferrato, Venise et Livourne, entre la fin du XVIII ${ }^{\mathrm{e}}$ siècle et les premières décennies du $\mathrm{Xx}^{\mathrm{e}}$ siècle. Ce corpus nous permettra d'émettre quelques propositions de lecture initiales et d'aborder la question de la conjonction des deux temps - le temps juif et le temps vulgaire - dans la vie sociale et culturelle des Juifs italiens. La variété des calendriers juifs publiés en Italie entre le $\mathrm{XvII}^{\mathrm{e}}$ siècle et le $\mathrm{XX}^{\mathrm{e}}$ siècle nous livre, en effet, un témoignage puissant de la nature du processus d'émancipation juive, fait d'avancées et de retours en arrière, et des transformations de l'identité juive italienne dans le passage, complexe et non linéaire, à la pleine jouissance de la citoyenneté. Une telle documentation d'usage populaire permet de repenser la relation entre Juifs et Chrétiens, le processus de reformulation des appartenances

* Davide Mano, Université de Strasbourg, membre du GEO et du CHER UR 4376.

1 La seule publication abordant la question des calendriers juifs en Italie en tant qu'objets de la culture populaire propose un plan descriptif de ces matériaux sans développer de piste d'analyse (Nahon 1966). 
sociales et politiques, ainsi que la réélaboration des cultures religieuses au tournant de l'Unité d'Italie (Ferrara degli Uberti 2011).

\section{L'organisation du temps dans le judaïsme: un excursus historique}

Il est essentiel d'amorcer quelques notions de base sur l'organisation du temps dans le judaïsme traditionnel, un sujet très complexe qui a stimulé de très longues discussions entre rabbins et intellectuels juifs et non juifs depuis l'antiquité. L'organisation du temps - à savoir, du temps du rite - devient un problème urgent dans la vie juive après la destruction du Second Temple de Jérusalem par les Romains en l'an 70 de l'ère commune. En effet, c'est après la perte de leur unité en tant que peuple et de leur souveraineté sur la Terre d'Israël, que les Juifs commencent à se poser le problème de l'unité du temps. Suite à l'altération du lien entre le temps rituel et le temps agricole en terre d'Israël, face aux nouveaux problèmes liés à la dispersion juive et à la consolidation d'une nouvelle vie juive dans des régions aussi éloignées que la Mésopotamie, l'Égypte, l'Italie ou l'Espagne, les maîtres rabbins décident de fixer des règles communes pour la détermination du calendrier juif (Stern 2001).

Une tradition juive antique raconte qu'autour de l'an 70, au cours du siège de Jérusalem par les Romains, Yohanan ben Zakkaï, chef du conseil des sages du Sanhédrin, recevait la permission de la part de l'empereur Vespasien de sortir de la ville et d'établir une académie de sages pharisiens à Yavneh, près de la côte méditerranéenne. En qualité de chef de ce nouveau conseil reconstitué loin de Jérusalem, Yohanan ben Zakkaï émettait des décrets urgents pour assurer le culte, prenait des décisions urgentes en ce qui concernait les dates des jours de fête, les débuts de mois, en recouvrant ainsi des tâches qui avaient été auparavant les prérogatives du grand prêtre du Temple et du Sanhédrin de Jérusalem (Neusner 1962; Mimouni 2012).

La nouvelle situation, sans temple, donc sans sacerdoce, demandait de repenser radicalement le culte juif. Toutefois, le lien avec la terre d'Israël devait être gardé autant que possible. Pendant les trois premiers siècles de diaspora juive, en effet, c'étaient encore des autorités rabbiniques attachées à la cour du Sanhédrin en terre d'Israël qui fixaient les dates du calendrier juif sur la base d'observations météorologiques, agricoles et astronomiques in situ.

Au IV ${ }^{e}$ siècle, cependant, un grand changement se produisit: après de longues études et des calculs très sophistiqués, le patriarche Hillel II décidait de renoncer à ces prérogatives en établissant des règles définitives pour le calcul du calendrier juif, applicables dans toute la diaspora. C'est très probablement à ce moment qu'était adopté le cycle métonique, ou lunisolaire, dans le judaïsme (Dickman 2009). L'autorité rabbinique établissait également que le calcul de l'an juif devait avoir comme point de départ la création du monde.

La condition diasporique des Juifs, marquée, entre autres, par la rupture du lien entre le temps du culte et le temps agricole, entraîne en effet des changements 
importants dans l'organisation du calendrier juif: en particulier, les maîtres rabbins établissent le «nouvel an» (Rosh Hashana), marquant l'anniversaire de la création du monde, à l'automne, au début du mois de Tishri, et non plus au début de la saison agricole, comme il avait été usage dans les temps bibliques (Jacobs 2007).

Ces décisions marquent la naissance d'un nouveau judaïsme, non seulement diasporique, sans temple ni terre, mais aussi sans autorité centrale: un judaïsme qui doit désormais se penser autrement, en Orient comme en Occident, et qui doit essayer de prospérer dans la diversité de ses diasporas, notamment par le biais de la fabrique communautaire, entendue comme lieu de rassemblement des dispersés. Naissant de la catastrophe de la destruction du Temple et de la perte de la souveraineté sur la terre d'Israël, ce judaïsme est désormais fondé seulement sur son unité de loi - la Torah - et de temps - le calendrier.

Le système du temps juif se fonde ainsi, depuis le $\mathrm{IV}^{\mathrm{e}}$ siècle, sur un système lunisolaire (Stioui 1988). Les mois sont lunaires - le deuxième jour du mois étant une nouvelle lune, tandis que les fêtes juives sont déterminées sur la base du système solaire (Amar 2019). Chaque mois se composant de 29 ou 30 jours, on peut calculer 11 jours de différence entre une année lunaire et une année solaire. C'est pour cette raison que le calendrier juif prévoit un treizième mois (ou mois embolismique) tous les deux ou trois ans. La semaine commence le dimanche et se termine le samedi, le shabbat, selon la succession temporelle de la création du monde relatée dans le livre de la Genèse (Gross 2015). Le jour commence à la tombée du soir (Sibony 1986).

Certes, aux premiers siècles de l'ère commune, en raison des changements traumatiques qui avaient bouleversé la vie juive, il était essentiel de fournir un système commun pour toutes les populations juives dispersées dans la diaspora, donc de fixer un calendrier universellement accepté par tous les groupes pratiquant le judaïsme. Un tel système commun aurait permis au peuple juif de garder une unité temporelle dans le culte, face à une condition de fragmentation géographique.

Le calendrier juif commun naît ainsi de la nécessité de fixer un système de calcul des années, des mois, des jours, partagé par tous les groupes juifs désormais éparpillés entre Orient et Occident. Il naît également de la nécessité de donner aux Juifs diasporiques un outil leur rappelant l'unité du peuple malgré leur dispersion physique. En découle le lien profond existant entre la conception du calendrier et la notion de mémoire dans le judaïsme (Spiegel 2002). Pareillement, l'objet calendrier définit sa nature et sa fonction en combinant dans la même page des éléments religieux, eschatologiques, saisonniers, historiques et nationaux: il véhicule une image essentiellement circulaire du temps (Goldberg 2000a).

Le temps constitue, donc, dans l'histoire de la diaspora du peuple juif, un élément unificateur essentiel. En effet, lorsqu'on s'attache à écrire l'histoire des Juifs, il est fondamental non seulement de restituer la dimension du 
polycentrisme juif, la constellation de centres et de cultures juives en Occident et en Orient, la diversité des espaces et des conditions dans lesquels la fabrique communautaire juive a pu se développer. Il est également indispensable de considérer ce macro-phénomène fondant la conscience historique juive, cet élément d'unité dans la dispersion qu'est le temps - et plus précisément le temps du culte, tel qu'il est organisé dans le calendrier juif, fondant la vision de soi du peuple juif en exil (Goldberg 1997). Dans le discours rabbinique et selon l'historiosophie juive traditionnelle, le temps est en effet un pont entre le passé et le futur: dans les jours de shabbat, comme dans les jours de fêtes, les Juifs réunis en collectif jouent une sorte de jeu de mémoire de souveraineté où la mémoire de leur «être-peuple» dans la terre d'Israël est ressuscitée. L'évocation des fastes des Hébreux bibliques comme expérience politique réveille ainsi l'idéal eschatologique: l'imagination est ainsi projetée dans le temps à venir, le temps de la délivrance et du retour, le temps du messie, le temps politique par excellence. Comme l'écrit Sylvie Anne Goldberg:

Les registres du temps ont une double vocation; ils se fondent au sein du temps du vécu pour former le cœur du principe de la vie juive. Transmission de la tradition et organisation du temps social se trouvent ainsi érigés au rang essentiel de la passation de la judéité. Si l'orientation de l'axe et de la flèche du temps revêt une importance déterminante, c'est que le temps est conçu comme la trame eschatologique par excellence. Tant que le temps ne semble pas être précisément défini par son terme, il n'est nul besoin d'en fixer la flèche. Dès lors que le temps est appréhendé comme ayant une origine conduisant à une finalité, sa linéarité pose que la création, origine du temps, parviendra immanquablement à son achèvement, qu'il consiste en un avènement messianique ou à la fin de son écoulement. (Goldberg 2000a)

\section{Une présence continue depuis l'antiquité : les Juifs d'Italie}

L'un des premiers lieux d'asile des Juifs en Occident fut l'Italie, et plus précisément l'Italie du centre et du sud. Si la communauté de Rome est la première communauté juive de la diaspora occidentale ( $\mathrm{I}^{\mathrm{er}}$ siècle EC), les premières présences juives dans la péninsule italienne datent, quant à elles, du $\mathrm{II}^{\mathrm{e}}$ siècle avant l'ère commune. Elles précèdent donc la destruction du Temple de Jérusalem et aussi la christianisation de la péninsule italienne. Les Juifs deviennent des citoyens de l'Empire romain au $\mathrm{III}^{\mathrm{e}}$ siècle EC et leur présence constitue une normalité dans une Italie multicentrique et multiculturelle lors des premières phases du processus de christianisation, allant du $\mathrm{IV}^{\mathrm{e}}$ siècle au $\mathrm{XVI}^{\mathrm{e}}$ siècle. Comme l'explique Giacomo Todeschini, du XVII ${ }^{\mathrm{e}}$ siècle à la fin du Moyen Âge, cette relative normalité devient progressivement une condition de minorité: la distance entre le regard chrétien et le regard juif se fait de plus en plus importante, devenant un facteur central vers la fin du Moyen Âge, lorsque la diffusion des stéréotypes antijuifs contribue à l'émergence d'un antijudaïsme de plus en plus virulent (Todeschini 2018). 
Pendant l'antiquité et une grande partie du Moyen Âge, la population juive est concentrée dans l'Italie du sud: les communautés démographiquement plus importantes se trouvent à Rome, dans les Pouilles, en Calabre, Campane et Sicile. Cette configuration de la présence juive est bouleversée en 1492 suite à l'expulsion des Juifs de Sicile, sous le contrôle de la couronne d'Espagne. Quelques décennies plus tard, un nouveau décret d'expulsion vise les Juifs du royaume de Naples. Plusieurs milliers de personnes sont ainsi forcées à quitter de manière définitive le sud de la péninsule italienne, pour s'établir dans le centrenord, à Rome, en Toscane, en Ombrie, à Bologne, à Venise, ou dans le Frioul. Dans un tournant contre-réformiste et particulièrement antijuif, la question de la gestion de ces nouvelles populations se pose alors au sein des pouvoirs dans les États italiens du centre-nord. La réponse est, toutefois, radicalement différente par rapport aux mesures prises dans les autres États chrétiens d'Europe: si en Angleterre, en France, en Allemagne et en Espagne les pouvoirs royaux et urbains ont opté, entre le $\mathrm{XIII}^{\mathrm{e}}$ et le $\mathrm{XV}^{\mathrm{e}}$ siècle, pour l'expulsion des Juifs de leurs territoires, dans les États italiens du centre-nord les Juifs sont contraints, à partir du $\mathrm{XVI}^{\mathrm{e}}$ siècle, à vivre dans des ghettos, des quartiers fermés et séparés du reste de la population des villes (Gasperoni 2016).

Une modalité d'exclusion - ou d'expulsion vers l'intérieur - est donc préférée à l'expulsion définitive : les Juifs doivent vivre séparément de la société chrétienne majoritaire, leur ségrégation - à savoir leur exploitation fiscale - devant servir au développement socioéconomique de ces États. Le premier ghetto est créé à Venise en 1516 pour des calculs éminemment économiques, tandis que le ghetto de Rome est établi en 1555 comme dispositif de conversion forcée des Juifs au catholicisme, servant par la suite de modèle pour la plupart des villes italiennes. L'histoire des réalités juives italiennes devient alors plus que jamais une histoire de résistance et de survie, d'adaptations et d'arrangements face aux mesures de minorisation juridique à leur encontre, face aux persécutions, aux pressions conversionnistes et à la précarité de la vie dans les ghettos (Gasperoni 2019; Mano 2017).

Ce système de gestion et contrôle des vies juives marque un premier pas d'arrêt vers la fin du $x$ III $^{\text {e }}$ siècle, lors de la première campagne d'Italie de Napoléon Bonaparte. Les ghettos sont abolis et les Juifs acquièrent la citoyenneté: c'est leur première émancipation, mais elle ne dure pas longtemps. Suite à la chute de l'Empire napoléonien, la plupart des États italiens restaurés rétablissent les mesures de ghettoïsation à l'égard des Juifs. La lutte pour l'émancipation se lie alors, à partir de 1848 , à la lutte pour l'unité nationale: les Juifs contribuent significativement à la cause du Risorgimento et leur émancipation complète coïncide avec l'Unité de l'Italie (Catalan 2012). Suite à la prise de Rome en 1870, les Juifs romains sont les derniers à sortir du ghetto.

Devenus des citoyens italiens à part entière, les nouveaux émancipés intègrent la société majoritaire, l'espace public, l'éducation et la culture nationale. Comme en France, leur nouvelle identité est exprimée par un nouveau terme collectif: ils ne se définissent plus comme des Juifs - terme d'ancien régime, associé au 
dictionnaire de l'antijudaïsme et à l'expérience du ghetto - mais comme des Israélites, c'est-à-dire des Juifs émancipés ou des citoyens de confession juive (Luzzatto Voghera 2004). Ce nouveau statut de citoyens à part entière restera en vigueur jusqu'en 1938, date de la promulgation des lois raciales par Benito Mussolini (Foa 2016).

\section{Vivre dans deux temps: les calendriers lunaires juifs italiens}

La documentation analysée dans le cadre de cet article est constituée d'une centaine de calendriers lunaires juifs (en italien Lunari ebraici), relevant des archives de la communauté d'Alessandria, dans le Piémont, et remontant à la période de la fin du Xvini ${ }^{e}$ siècle aux premières décennies du $\mathrm{XX}^{\mathrm{e}}$ siècle ${ }^{2}$. Il s'agit de petits almanachs au format in-32 et aux couvertures coloriées avec des motifs floraux, qui étaient en usage dans les communautés juives italiennes. Imprimés à Turin, à Casale Monferrato, à Livourne ou à Venise, ils avaient servi la grande majorité des communautés juives d'Italie, des communautés situées en milieu urbain comme rural. Ils avaient été conçus pour une très large diffusion et selon des modèles éditoriaux assez simples et rudimentaires: la mise en page reflétait une finalité de praticité et simplicité, mais aussi la nécessité de produire un outil à bas prix, accessible à tout le monde. Leur usage était censé être très populaire: ils devaient fournir un outil pratique pour tout agriculteur ou éleveur, pour tout marchand et voyageur juif, ayant besoin d'un instrument du monde, sorte de boussole pouvant répondre à ses besoins et aussi refléter sa vie. À cet effet, ils proposaient des informations générales, notamment astronomiques: les phases de la lune et la durée des jours y étaient indiquées, ainsi que des informations statistiques ou encore des calculs plus strictement religieux, comme par exemple les horaires précis d'entrée des shabbatot.

Ces calendriers lunaires juifs italiens reprenaient tant les grandes dates du calendrier juif que les dates du calendrier chrétien ou vulgaire - vulgaire étant l'adjectif pour indiquer ici les goyyim, en hébreu les "populations» non juives majoritaires, sans acception péjorative. Ces objets, dans lesquels le temps juif et le temps chrétien coexistaient, avaient été imprimés en caractères latins pour rejoindre la population moins cultivée. Au XVIII siècle, la population juive était presque totalement alphabétisée, un élément qui la différentiait significativement de la population chrétienne qui comptait encore un nombre non négligeable d'analphabètes. Cependant, la maîtrise de l'hébreu s'était affaiblie au sein des communautés, ce qui avait entraîné un usage de plus en plus fréquent de la langue vernaculaire, l'italien, avec ses spécificités régionales. En outre, ces petits almanachs récupéraient tout probablement des modèles de presse déjà existants, afin d'assurer des coûts de production très bas et ainsi garantir le maintien d'un prix populaire. Les débuts des mois, les samedis et les jours de fête présentaient

2 Archivio ebraico Terracini, Comunità, Comunità Ebraica di Alessandria (I Versamento), 9. Periodici a stampa e varie, 9.1. Lunari, 1790-1931. 
certes des informations juives, mais très succinctes en raison du manque d'espace; le plus souvent, les mots hébreux étaient translittérés en caractères latins pour faciliter au maximum la préparation technique de la page.

Dans l'histoire du livre imprimé, l'almanach joue certes un rôle important en tant que moyen de vulgarisation des savoirs. À partir du $\mathrm{Xv}^{\mathrm{e}}$ siècle et jusqu'au $\mathrm{xx}^{\mathrm{e}}$ siècle, il constitue une lecture très populaire dans toute l'Europe et figure parmi les ouvrages les plus imprimés et les plus lus de la population moins privilégiée, occupée pour la plupart dans le travail agricole et artisanal. Outre les saints du jour et les prévisions météorologiques, bien utiles tant pour les agriculteurs que pour les navigateurs, le petit carnet pouvait contenir les prix des récoltes et des bétails, les dates et les lieux des foires et de marchés agricoles les plus importants, ou encore des tables astronomiques déterminant, pour chaque jour ou semaine ou mois, les positions des planètes, de la lune, du soleil, des étoiles (Casali 2003). On pouvait y repérer également des rudiments de médicine, des savoirs traditionnels, des conseils horticoles. Des contes, des proverbes, des brèves ou des curiosités historiques, rédigés pour tout public et dans un style populaire, offraient enfin un dérivatif agréable aux fatigues du travail quotidien. $\mathrm{Au} \mathrm{XVIII}^{\mathrm{e}}$ siècle, ces éphémérides étaient devenues de véritables outils de diffusion culturelle, parmi les populations rurales notamment (Braida 1989).

En ce qui concerne le contexte juif, ces objets de la culture populaire s'inscrivaient dans un univers d'œuvres de vulgarisation, pour la plupart en langue vernaculaire, combinant le savoir religieux au politique et à l'économique, le particularisme juif au sentiment d'une appartenance plus large et aux besoins pratiques de la (sur)vie. Les calendriers lunaires juifs reflétaient directement la réalité sociale et culturelle des Juifs, leur condition de minorité religieuse au sein de la chrétienté. Ils représentaient sur la page l'équilibre difficile entre l'intégration à la société dominante et la résistance culturelle et religieuse de la minorité, la coexistence instable entre l'acculturation aux modèles de vie majoritaire et la détermination à perpétuer son héritage particulier (Zerubavel 1981). Le calendrier exprimait, en effet, la nécessité générale pour tout Juif de savoir vivre dans les deux mondes, celui de la communauté religieuse et celui de la société environnante. Pour persister dans la diaspora, tout Juif avait besoin de connaître et de maîtriser les deux systèmes du temps, celui du judaïsme et celui de la chrétienté. Il lui était également demandé de s'exprimer dans les deux langues - l'hébreu et l'italien - et de bien connaître les deux cultures juridiques - le droit rabbinique d'une part, et le droit canon et le droit commun d'autre part. En tant que paradigme de la minorité en Europe, les Juifs étaient censés savoir vivre dans les deux systèmes culturels, sociaux, politiques, juridiques et économiques, pour pouvoir à la fois pérenniser leur spécificité culturelle et survivre à leur condition de minorisation. Connaître le calendrier religieux des chrétiens leur était absolument indispensable, car cela leur permettait d'acquérir un savoir utile pour mieux interagir avec le pouvoir ou mieux échanger avec leurs voisins (Mano 2020). Ce savoir sur l'autre n'était bien évidemment pas demandé aux chrétiens, membres de la communauté sacrée de la chrétienté, qui 
en revanche savaient bien peu de choses sur les Juifs, sur leurs coutumes, et qui le plus souvent tiraient leurs références d'une tradition de stéréotypes antijuifs créés dans le but de perpétuer la méfiance à l'égard des Juifs.

\section{L'organisation des calendriers juifs italiens au tournant révolutionnaire}

La question de la connaissance de l'autre est particulièrement importante lorsqu'on aborde une période de profondes transformations comme celle de la transition révolutionnaire et du premier processus d'émancipation juive. De la fin du $\mathrm{XVIII}^{\mathrm{e}}$ siècle au début du XIX ${ }^{\mathrm{e}}$ siècle, les calendriers lunaires juifs italiens montrent des évolutions notables en ce qui concerne tant l'autoreprésentation du groupe que l'organisation des deux temps juif et chrétiens, et plus en général des savoirs (Goldberg 2000b, Neusner 1990).

Un premier exemple est constitué par le «Calendrier lunaire de l'an 1793 et 5553 de la création du monde selon le calcul des Juifs », dans lequel sont indiqués les «fêtes mobiles et immobiles» de Juifs et chrétiens, les «quatre temps» du jour, le «levant» du soleil, les "vraies nouvelles lunes et pleines lunes selon le calcul des Juifs» et "les fêtes, les jeûnes, les solennités, et les jours des mois lunaires selon les Juifs». Il s'agit d'un almanach composé par Salomon Moise Bachi de la communauté juive de Casale Monferrato, dans le Duché de Savoie, à la veille de la première campagne napoléonienne en Italie. Les informations astronomiques sur le "point où le soleil entre dans les signes de l'Écliptique, le midi et le minuit dans chaque mois » y occupent une place importante.

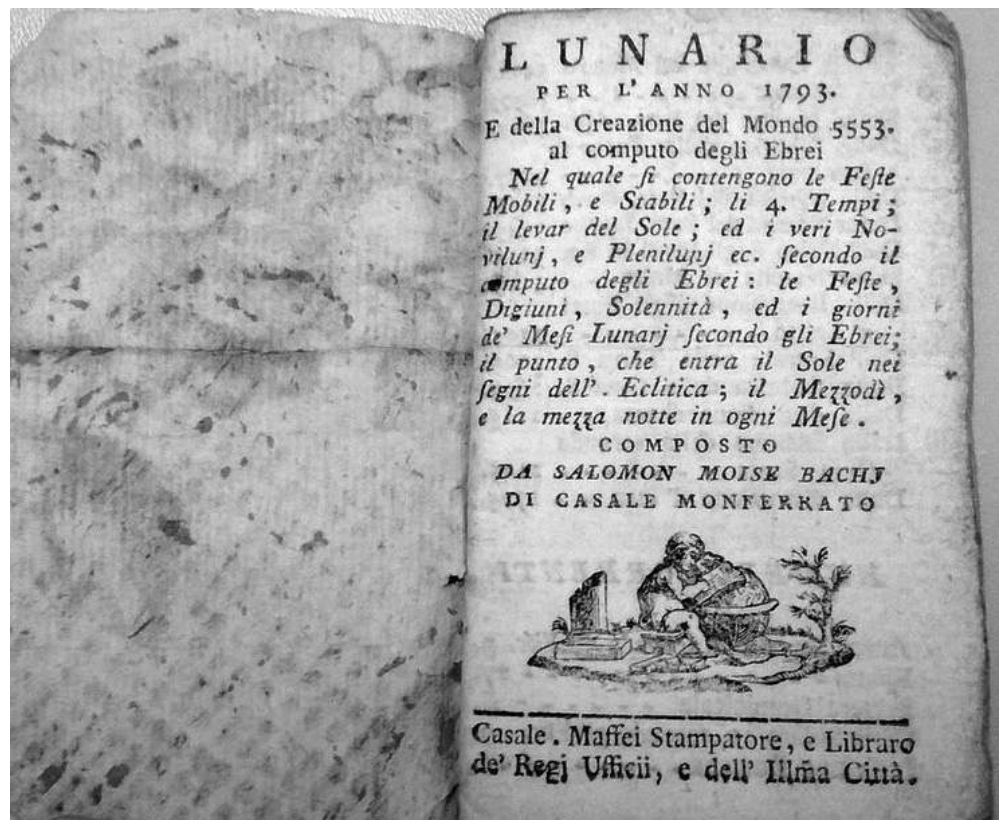

Calendrier lunaire de l'an 5553 (1793), @ Archivio ebraico Terracini, Comunità, Comunità Ebraica di Alessandria (I Versamento), 9. Periodici a stampa e varie, 9.1. Lunari, 1790-1931. 
Un autre exemple prérévolutionnaire est celui du «Calendrier lunaire pour l'an 1794 et 5554 de la création du monde selon le calcul des Juifs» imprimé à Venise, qui offre une profusion d'informations pratiques générales concernant les temps de la vie économique et institutionnelle vénitienne. Il informe, entre autres, des « ouvertures et fermetures du Banco Giro ", à savoir la banque publique de Venise située à Rialto, ainsi que de «l'heure de Tierce pour le Foro Veneto", à savoir des heures de réception du tribunal vénitien. Il donne également un aperçu des «mois des Turques et leurs festivités», très probablement dans le but de faciliter les commerces avec des marchands musulmans.

Un troisième exemple, le «Calendrier lunaire juif pour l'an 5558 et selon l'ère vulgaire 1798 ", imprimé à Turin par les soins d'Abram Salomon Verona, montre des nouveautés évidentes par rapports aux deux exemples précédents: l'adjectif "juif»(ebraico en italien) apparaît dans le titre et l'année juive est indiquée avant la date vulgaire. Une table d'histoire sacréejuive est également présentée dans les premières pages avant le calendrier proprement dit : il s'agit d'une succession d'évènements inscrits dans l'histoire juive allant du plus ancien au plus récent. On peut y lire: «5558 ans depuis la création du monde, 3902 depuis le déluge, 3610 depuis la naissance du patriarche Abraham », et par la suite «2630 ans depuis l'édification du premier Temple de Jérusalem» et «1730 ans depuis la destruction du Second Temple».

Ces trois calendriers de fin $\mathrm{XVIII}^{\mathrm{e}}$ siècle présentent une organisation du temps suivant le modèle du calendrier vulgaire: ils commencent tous au $1^{\text {er }}$ janvier. Pour chaque jour est indiqué le nom du saint chrétien, les solennités principales des deux religions étant signalées en italique. Pour chaque shabbat, le nom de la portion de lecture biblique est présenté en translittération en caractères latins. Des notes succinctes précisent les temps de la pleine lune et, à chaque vendredi, les heures d'entrée du shabbat.

Le «Calendrier lunaire juif pour l'an 5559, 1799 selon l'ère vulgaire et $7^{\mathrm{e}}$ an de la République française», imprimé à Turin par les soins d'Abram Salomon Verona, nous emmène au cœur de la première période révolutionnaire italienne, datant de 1796 à 1799. Cet exemplaire offre, comme d'habitude, des informations astronomiques utiles accompagnées d'autres indications de nature cultuelle: l'ordre des lunes "selon le méridien turinois», les horaires d'entrée du shabbat chaque vendredi soir, mais aussi le «journal des saints à usage des catholiques». Quelques nouveautés importantes y sont visibles : l'almanach présente d'abord trois colonnes pour la succession des jours selon les trois calendriers: juif, vulgaire et révolutionnaire. Des phases du calendrier révolutionnaire sont ajoutées aux informations concernant les solennités chrétiennes et juives: une succession en décades est consacrée à des valeurs républicaines, telles que la vérité et la justice. 

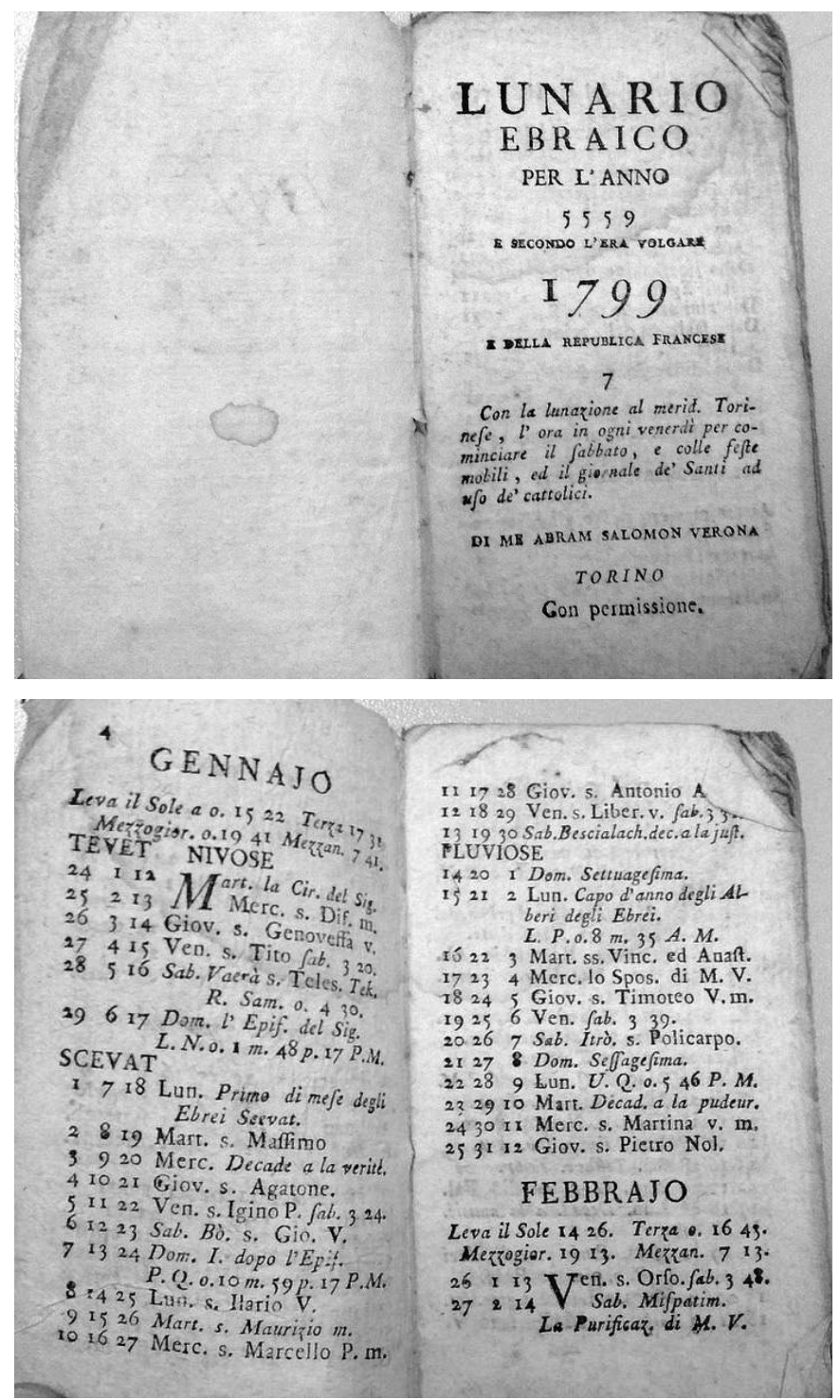

Calendrier lunaire de l'an 5559 (1799), @ Archivio ebraico Terracini, Comunità, Comunità Ebraica di Alessandria (I Versamento), 9. Periodici a stampa e varie, 9.1. Lunari, 1790-1931.

La première saison révolutionnaire italienne s'achevant très tôt, le calendrier lunaire juif pour l'an 1800 revient sur la dualité des temps chrétien et juif. Une nécessité de marquer une distinction entre les deux religions dans l'espace de la page, déjà très étroit, de l'almanach, semble ici se manifester: la nouveauté majeure se trouve en effet dans l'usage du signe d'une croix pour indiquer les fêtes catholiques et d'un astérisque pour indiquer les fêtes juives. 
Si l'on considère un exemplaire du calendrier lunaire juif de «l'an 5564 et 1803-1804 selon l'ère vulgaire», an «bissextile» et «le $12^{\text {e }}$ de la République française", imprimé à Turin, l'on constate, avec le retour de la domination française, la réapparition des trois systèmes du temps. Une table des chronologies sacrées, ainsi que les dates des fêtes mobiles chrétiennes et juives, précèdent les informations astronomiques, concernant entre autres deux éclipses de soleil et deux de lune, prévues pendant l'année. La grande nouveauté dans ce cas est que le calendrier ne commence pas le $1^{\text {er }}$ janvier mais au nouvel an juif, le $1^{\text {er }}$ jour du mois de Tishri, correspondant ici au 30 du mois de Fructidor et au $17 \mathrm{du}$ mois de Septembre. C'est un changement très significatif dans l'organisation de l'almanach: la question se pose ici de savoir s'il existe une corrélation entre le nouveau cadre politique, celui de la deuxième tutelle républicaine française sur le territoire de l'ancien Duché de Savoie, et ce changement dans l'organisation du calendrier lunaire, qui semblerait bouleverser l'ordre établi en privilégiant le système du temps juif. Une telle modification semblerait justement vouloir témoigner de l'importance du changement politique en cours.

Quant aux années sous l'Empire napoléonien, l'«Almanach oriental illustré pour l'an 1812, bissextile, et 5572 depuis la création du monde», imprimé à Livourne par les soins de Raffaello David Meldola, commence aussi à partir du 19 septembre 1811, date qui correspond au nouvel an juif, selon le calcul des "Israélites». La nouvelle terminologie de l'émancipation à la française apparaît ici comme acquise. Dans cet exemplaire, on trouve beaucoup de nouveautés par rapport aux calendriers présentés jusqu'ici: on y repère des légendes des lectures de la Torah prévues pour chaque samedi de l'an juif, plusieurs chapitres bibliques, tirés des livres des Prophètes ou des Psaumes, ainsi qu'un tableau des naissances et des anniversaires des souverains impériaux et royaux de France et d'Italie. La chronologie sacrée s'enrichit notablement: on y trouve mentionnés les «783 ans depuis la naissance du célèbre Rashi, 679 depuis la naissance du célèbre Maimonide», ou encore "362 ans depuis la création de la presse à Mayence, 8 depuis le couronnement impérial de notre Souverain». Une description des fêtes israélites est jointe par la suite, ainsi qu'une table des horaires d'entrée du shabbat et un aperçu du calendrier "des Turques». Une autre innovation y est visible: une page est entièrement consacrée à la "Cabale» pour les amateurs du jeu de la Loterie impériale de France. Le calendrier lui-même commence à la date du $1^{\mathrm{er}}$ janvier, mais les jours ne sont plus accompagnés des noms des saints catholiques, ni des fêtes chrétiennes, mais exclusivement des noms des solennités juives et autres informations astronomiques et météorologiques. Des jours de commémoration locale (livournaise) sont aussi notés, comme le jour de l'anniversaire du tremblement de terre de l'an 1771 en date du 7 janvier. 

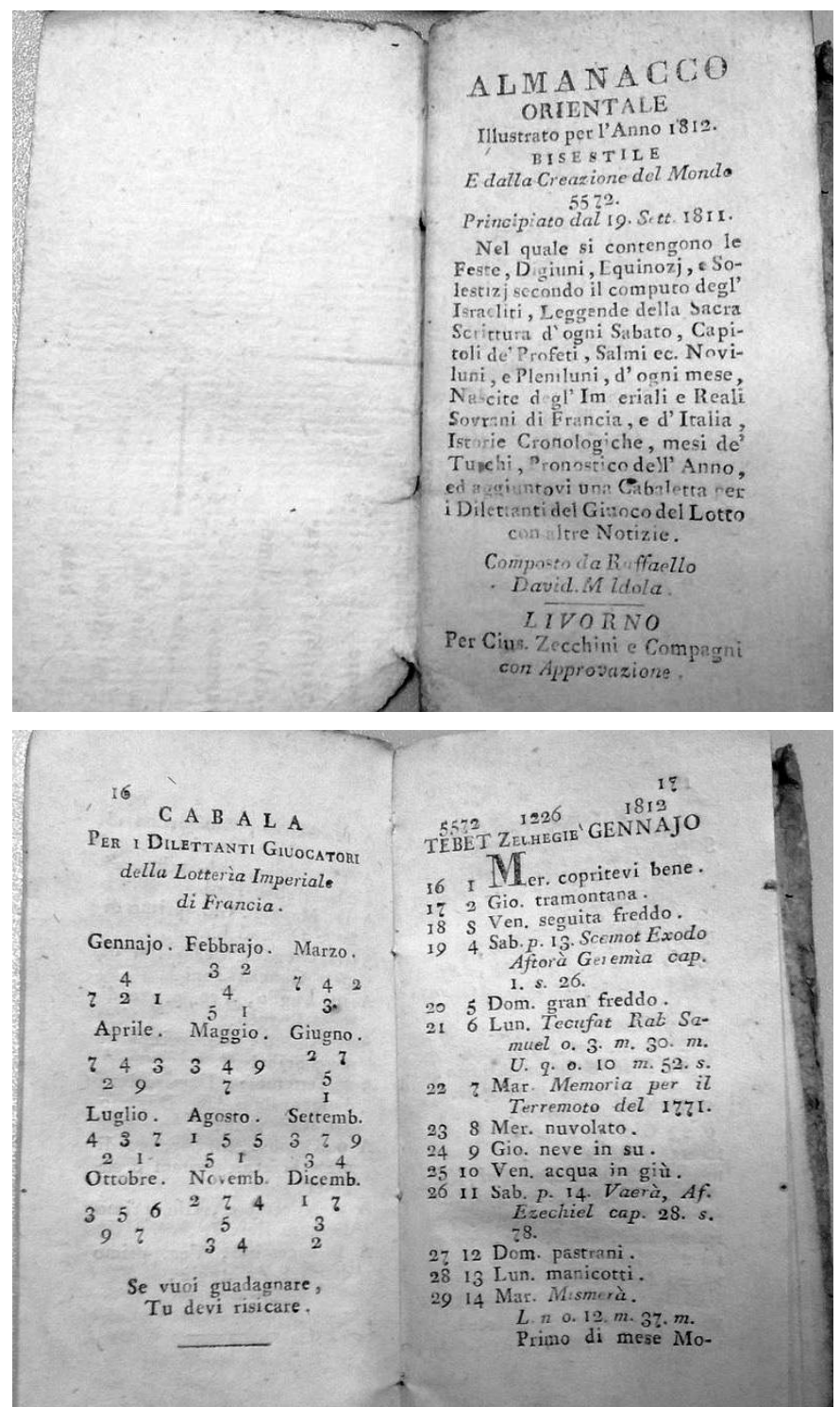

Almanach oriental de l'an 5572 (1812), @ Archivio ebraico Terracini, Comunità, Comunità Ebraica di Alessandria (I Versamento), 9. Periodici a stampa e varie, 9.1. Lunari, 1790-1931.

L'Almanach oriental illustré est relié à un deuxième calendrier lunaire livournais «à l'usage des Florentins» où l'on trouve notamment l'indication des fêtes de l'Empire français, dont la fête de Saint Napoléon le 15 août (à la même date que l'Assomption), ou encore les fêtes pour l'anniversaire du couronnement de Sa Majesté Impériale et de la Bataille d'Austerlitz, tombant le premier dimanche de décembre. 
Dès 1815, la période de la Restauration nous offre d'autres exemples intéressants, qui montrent des continuités et des discontinuités par rapport aux calendriers de l'époque révolutionnaire et napoléonienne. Le «calendrier lunaire juif pour l'an du monde 5588» (1827-1828), imprimé à Turin par Rafael Uri Foa, poursuit le modèle de l'Almanach oriental livournais. La table chronologique présentée dans ses premières pages comprend de nouvelles dates historiques relevant du calendrier local, dont la mention des « 122 ans depuis la libération de Turin du siège de 1706 », des « 7 ans depuis le royaume de Sua Sacra Real Maestà Carlo Felice» et des « 4 ans depuis la création du Collège israélite à Turin». On y trouve également une page entièrement consacrée aux dates de naissance de la famille royale. Le calendrier commence par le premier jour de l'an juif, mais les saints catholiques sont réintroduits. La nouveauté majeure réside dans le fait d'inclure de plus en plus de textes annexes, dont des traités rabbiniques en traduction italienne.

\section{L'heure de l'émancipation: une renaissance sociale, culturelle et spirituelle}

L'unification de l'Italie entre 1861 et 1870 constitue un véritable tournant dans le processus d'émancipation juive. Pour les Juifs italiens, l'acquisition de la citoyenneté complète n'est pas seulement synonyme d'intégration et d'assimilation à la société majoritaire: l'émancipation constitue également une occasion de renaissance juive, entraînant la redécouverte de sa culture particulière dans un contexte de liberté, le retour aux sources et la renaissance de la langue des ancêtres (l'hébreu). L'émancipation est ainsi l'occasion de la revitalisation du sentiment d'identité juive, de la réélaboration du judaïsme comme système de croyances et rites en parfaite conformité avec l'Etat, la démocratie et la modernité. Les calendriers lunaires juifs italiens de la deuxième moitié du XIX ${ }^{\mathrm{e}}$ siècle permettent de voir cette convergence - une dynamique à la fois d'assimilation politique et de renaissance culturelle - de manière particulièrement claire. Des modifications très significatives sont apportées dans la mise en page, dans l'organisation des savoirs, dans l'usage des langues, dans la hiérarchie des informations religieuses et non, dans l'esthétique.

Le «calendrier lunaire juif» de 5623 (1863), imprimé à Venise, présente une première nouveauté: dans la première de couverture, l'année est présentée aussi en caractères hébraïques. Il est précisé également qu'elle se compose de 354 jours, dont 51 samedis. L'ère vulgaire, quant à elle, y est désignée comme "ère chrétienne». Pour la première fois, on observe une information à caractère commercial: la formule " en vente à la foire de Sinigaglia» nous informe de la large diffusion dont cet almanach jouissait parmi les marchands et les entrepreneurs agricoles.

Le «calendrier lunaire israélite pour l'an de la création 5644 »(1883) imprimé à Casale Monferrato, par le chef-rabbin Ferruccio Servi, montre davantage de nouveautés: sur la première de couverture, l'année vulgaire est présentée en caractères latins tous petits et entre parenthèses, tandis qu'une citation en hébreu 
tiré de Psaumes 84 occupe une place plus importante. Au verso de la première de couverture, l'on rencontre les premières publicités de livres à sujet juif en vente chez le même éditeur du calendrier. Le chef-rabbin Ferruccio Servi est désigné par son titre de chevalier du Royaume d'Italie et en qualité de directeur de la revue «Il Vessillo Israelitico» de Turin, l'une des premières revues juives italiennes à sujet historico-littéraire (Ferrara degli Uberti 2007).

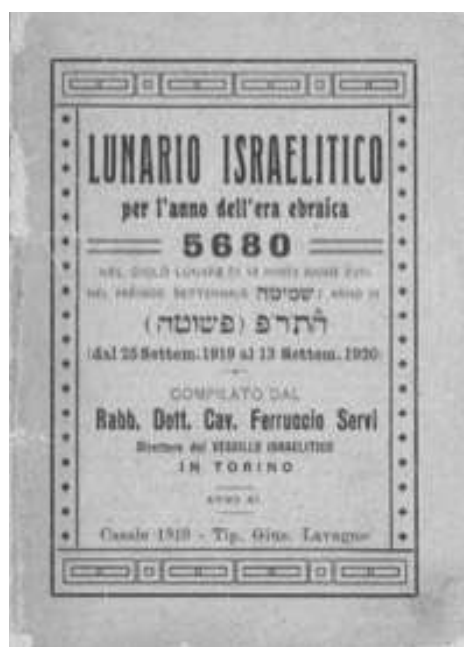

Calendrier lunaire de l'an 5680 (1919-1920), @ Archivio ebraico Terracini, Comunità, Comunità Ebraica di Alessandria (IVersamento), 9. Pe riodici a stampa e varie, 9.1. Lunari, 1790-1931.

Encore plus intéressante est la première de couverture du «Calendrier lunaire israélite de l'an 5680 de l'ère juive» (19191920), compilé par le même rabbin Ferruccio Servi et imprimé à Casale Monferrato. Dans ce cas pour la première fois, l'on trouve la concomitance des termes «israélite» et "juif». L'émancipation ayant entraîné un retour massif des Juifs à la terre, l'on constate une attention renouvelée aux dispositions bibliques et rabbiniques concernant l'organisation du temps agricole: on précise, en effet, que l'année en question correspond à «la dix-septième année dans le cycle lunaire de 19 ans» et à "la troisième année» dans le cycle de sept ans de la shemitah. Ce dernier mot, inscrit en caractères hébraïques, est une référence au temps agricole dans la Bible, la shemitah étant l'année de jachère que les agriculteurs juifs doivent observer tous les sept ans selon les prescriptions bibliques.

Dix ans plus tard, le calendrier lunaire israélite de 5691 (1930-1931) présente enfin une annonce commerciale sur la première de couverture: une publicité du bitter Campari de Milan. En bas de page, l’on remarque également la mention du prix de l'almanach («L. 2,50»). À l'intérieur, d'autres publicités trouvent leur place, comme celle présentée sur le verso de la première de couverture: l'annonce d'un marchand et agent financier du Caire, en Égypte, avec des filières en Palestine, Syrie et Turquie. Si l'édition de ce calendrier est désormais faite par les soins de la «librairie F. Servi» de Turin, l'une de ses nouveautés les plus remarquables est la table démographique des Israélites d'Italie présentée dans les premières pages : pour chaque ville il est indiqué le numéro des résidents juifs, membres inscrits dans la communauté locale, ainsi que le nom du rabbin en service. Aussi les communautés juives de Lybie, à l'époque une colonie italienne, y sont comptées.

La pagination est quant à elle complètement renouvelée: la page de gauche est pour le calendrier juif avec une présence toujours plus importante d'informations en hébreu, tandis que la page de droite est pour le calendrier dit «italien». À gauche, les informations concernent exclusivement le culte, 
tandis qu'à droite presque chaque jour est accompagné d'un anniversaire dans l'histoire juive: par exemple, le 27 septembre coïncide avec "l'anniversaire de l'émancipation des Juifs de France en 1791 », et le jour avant est l'anniversaire de la «naissance de Moise Mendelssohn à Dessau en 1729». Cette version du calendrier israélite n'est pas seulement entièrement déchristianisée, elle offre aussi une place de plus en plus importante au savoir historique, considéré comme le représentant du nouvel ethos israélite.
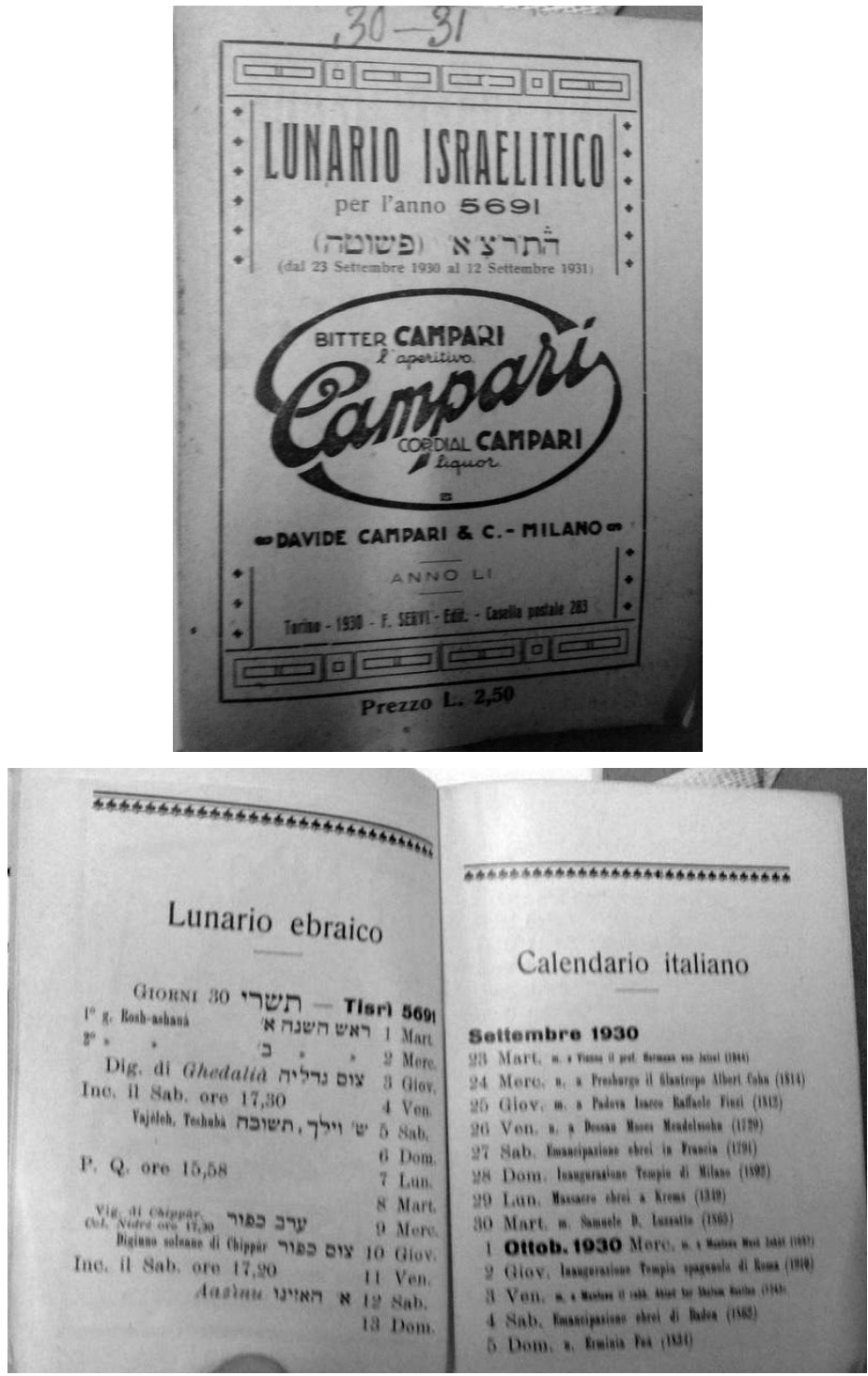

Calendrier lunaire de l'an 5691 (1930-1931), @ Archivio ebraico Terracini, Comunità, Comunità Ebraica di Alessandria (I Versamento), 9. Periodici a stampa e varie, 9.1. Lunari, 1790-1931. 
Nous sommes seulement sept ans avant la promulgation des lois raciales et racistes du fascisme italien, évènement qui amènera à l'exclusion des Juifs de l'espace public, des écoles, des universités, des bureaux, des arts. Un appareil d'Etat minutieux sera ainsi mis en place pour exécuter l'exclusion des Juifs de la société italienne pour cause raciale. La publication des calendriers lunaires juifs s'arrêtera pendant les années de la Seconde Guerre mondiale. Elle reprendra dans l'immédiat après-guerre, en Italie comme dans le reste d'Europe, lorsque les calendriers récupèreront leur fonction sociale et culturelle au sein du monde juif.

\section{Conclusions}

Dans le long processus d'émancipation juive en Italie, un processus marqué par des avancées et des retours en arrière, les changements dans la conception et la fabrication des calendriers lunaires juifs ont été nombreux. On peut constater, de manière générale, un passage progressif d'un calendrier sur le modèle de l'almanach chrétien à un calendrier de plus en plus défini par son caractère juif, où la place de l'hébreu se fait importante. Cette évolution ne serait pas à interpréter comme un acte de repli sur soi au moment de l'émancipation, justifié par le risque d'assimilation, mais elle serait à comprendre plutôt comme un acte d'appropriation juive de la notion d'émancipation: à l'émancipation des droits, à l'entrée dans la citoyenneté, l'on faisait suivre, par nécessité de cohérence, une émancipation culturelle et spirituelle. L'émancipation signifiait, enfin, la liberté de pouvoir prospérer dans le bain de sa culture d'origine, outre le bain de la culture nationale. Elle consentait à reformuler l'identité juive par le biais d'une ouverture au savoir scientifique, notamment historique. L'émancipation devenait ainsi le synonyme tant d'une ouverture complète et heureuse vers la société italienne que d'une redécouverte de son identité juive, alias israélite.

\section{Bibliographie}

Amar R.B., 2019, Sull'osservanza delle feste. Descrizione delle festività, digiuni e giorni distinti di culto ebraico nel corso dell'anno, sous la direction de A. M. Somekh, Livorno, Belforte editore.

Braida L., 1989, Le guide del tempo. Produzione, contenuti e forme degli almanacchi piemontesi nel Settecento, Torino, Deputazione subalpina di storia patria.

Carlebach E., 2011, Palaces of Time: Jewish Calendar and Culture in Early Modern Europe, Cambridge (MA), Belknap Press.

Casali E.,2003, Le spie del cielo. Oroscopi, lunari e almanacchi nell'Italia moderna, Torino, Einaudi.

Catalan T., 2012, «Les juifs italiens et le Risorgimento: un regard historiographique», Revue d'histoire du XIX ${ }^{e}$ siècle, 44, p. 127-137. 
Dickman B., 2009, «The Beginning of the Jewish Calendar», Hakira, 8, p. 223-235.

Ferrara degli Uberti C., 2007, "Rappresentare se stessi tra famiglia e nazione. Il 'Vessillo Israelitico' alla soglia del '900», Passato e presente, 70, p. 35-58.

Ferrara degli Uberti C., 2011, Fare gli ebrei italiani: autorappresentazioni di una minoranza, Bologna, Il Mulino.

Foa A., 2016, «Les Juifs italiens entre le Risorgimento et le fascisme: un regard d'ensemble», Revue d'Histoire de la Shoah, 204, p. 19-34.

Gasperoni M., 2016, «1516, Naissance des ghettos», L'Histoire, 427, p. 13-19.

Gasperoni M., 2019, dir., Le siècle des ghettos: la marginalisation sociale et spatiale des juifs en Italie au XVII ${ }^{e}$ siècle, Revue du XVII siècle, 282, Janvier-Mars 2019.

Goldberg S. A., 1997, «De la Bible et des notions d'espace et de temps. Essai sur l'usage des catégories dans le monde achkénaze du Moyen Âge à l'époque moderne», Annales HSS, 5, p. 987- 1015.

Goldberg S. A., 2000, La Clepsydre. Essai sur la pluralité des temps dans le judaïsme, Paris, Albin Michel.

Goldberg S. A., 2000a, "Contes et décomptes du temps juif», Bulletin $d u$ Centre de recherche français à Jérusalem, 7, <http://journals.openedition.org/ bcrfj/2332>.

Goldberg S. A., 2000b, «Questions of times: Conflicting time scales in historical perspective», Jewish History, 14, p. 267-286.

Goldberg S. A., 2004, La Clepsydre II. Temps de Jérusalem, temps de Babylone, Paris, Albin Michel.

Gross B., 2015, Shabbat, Paris, Éditions de l'Éclat.

Jacobs L., 2007, "Rosh Ha-Shanah», Encyclopaedia Judaica, vol. 17, 2e édition, sous la direction de Michael Berenbaum et Fred Skolnik, Detroit, Macmillan Reference, p. 463-466.

Laras G., 2015, La natura del pensiero ebraico. L'origine del mondo e i concetti di spazio e di tempo, Milano, CUEM.

Luzzatto Voghera G., 2004, «L'israélitisme en Italie aux XIX et $\mathrm{XX}^{\mathrm{e}}$ siècles », dans Cabanel P. et Bordes-Benayoun Ch. (dir.), Un modèle d'intégration. Juifs et israélites en France et en Europe (XIX ${ }^{e}-\mathrm{XX}^{e}$ siècles), Paris, Berg International, p. 197-207.

Mano D., 2017, «L'Italie et «ses» Juifs: modernités, mobilités, identités », En Jeu. Histoire et mémoires vivantes, 9, p. 27-39.

Mano D., 2020, "The Notion of Reciprocity and the Logics of Belonging. An Inquiry into Jewish and Christian Petitions from Pitigliano (18 $8^{\text {th }}$ century)», in Guetta A. et Savy P. (dir.), Non contrarii, ma diversi. The Question of the Jewish Minority in Early Modern Italy, Roma, Viella, p. 213-224.

Mimouni S. C., 2012, Le judaïsme ancien $d u$ vI siècle avant notre ère au III $^{e}$ siècle de notre ère, Paris, PUF.

Nahon U., 1966, «Il lunario di Venezia », La Rassegna Mensile di Israel, 8, vol. 32, p. 365-378. 
Neusner J., 1962, A Life of Rabban Yohanan ben Zakkai (Ca. 1-80. C.E.), Leiden, Brill, Studia Post-Biblica.

Neusner J., dir., 1990, The Christian and Judaic Invention of History, Atlanta, AAR 55, Scholars Press.

Sibony M., 1986, Le jour dans le judaïsme, Son histoire et ses moments significatifs, Tours, Faculté des sciences.

Spiegel G. M., 2002, "Memory and History: Liturgical Time and Historical Time», History and Theory, 2, vol. 41, p. 149-162.

Stern S., 2001, Calendar and Community. A History of the Jewish Calendar, 2nd Century BCE to 10th Century CE, Oxford, Oxford University Press.

Stioui R., 1988, Le calendrier hébraïque, Paris, Colbo.

Todeschini G., 2018, Gli ebrei nell'Italia medievale, Roma, Carocci.

Zerubavel E., 1981, Hidden Rythms. Schedules and Calendars in Social Life, Chicago, University of Chicago Press. 